\title{
Investigation of Nonlinear Multi-connected Systems of Automatic Control with Delay
}

\author{
Baryi Ilyasov \\ Department of technical Cybernetics \\ Ufa stste aviation technical university \\ Ufa, Russia \\ ilyasov@tc.ugatu.ac.ru
}

\author{
Guzel Saitova \\ Department of technical Cybernetics \\ Ufa stste aviation technical university \\ Ufa, Russia \\ saitova@bk.ru
}

\author{
Anastasia Elizarova \\ Department of automatic control \\ system \\ Ufa stste aviation technical university \\ Ufa, Russia \\ elizarovaanastasia@gmail.com
}

\begin{abstract}
The problem of controlling objects with time delay is quite complicated. The presence of a delay in the control loop leads to an increase in the phase shift, which can provoke instability of the system. The paper proposes a study of a nonlinear multi-connected system of automatic control (MSAC) of an object with a delay by frequency methods, based on the method of harmonic linearization and a system description of the characteristics of a multi-connected automatic control system. A nonlinear multi-connected automatic control system with delay in direct channels and cross-channels is considered. The nonlinear multi-connected automatic control system with delay consists of a set of identical separate subsystems and mutual connections between subsystems are single. With the help of frequency methods, the parameters of periodic motions (PM) in nonlinear homogeneous multi-connected automatic control systems with delay (amplitude and frequency of self-oscillations) are determined and their stability is estimated according to the proposed criteria. The paper presents examples that demonstrate the use of the proposed techniques to assess the stability of a nonlinear multi-connected system of automatic control of the object with delay both in direct communication channels and cross and finding the parameters (amplitude and frequency) of periodic movements. The results of the study were confirmed by simulation using MATLAB SIMULINK package.
\end{abstract}

Keywords-multi-connected systems of automatic control, delay, nonlinear element, periodic motion, frequency method, stability.

\section{INTRODUCTION}

In many modern technical objects of control (OC) can observe the phenomenon of delay, consisting in the fact that with the beginning of the signal change at the input of the OC, signal at the output of the object begins to change only after a certain interval of time. For example of control objects with delays can be jet engines in transient modes, belt conveyors, rolling mills, drying and combustion processes, on a state - processes with a recycle, in particular, processes in grinding machines or processes in chemical reactors, at the output - control objects with more inertial measurement sensors than the object itself $[1,14]$.

Generally, the influence of delay on the management processes is negative. Therefore, methods of studying control systems for the above objects, not considering the delay factor, are ineffective. The problem of designing similar control systems is even more complicated if the control object is multi-connected and nonlinear.

We know a large number of works, devoted to investigate of automatic control systems (ACS) with delay on control with one input and one output . A small number of scientific papers are devoted to multidimensional (multi-connected) control systems with delays [2].

In article proposes a study of a multi-connected nonlinear system of automatic control of the object with delays (control and output) by frequency methods, based on the method of harmonic linearization and system description of the characteristics of the MSAC.

\section{RESULTS OF INVESTIGATION}

We will propose in the future that this class of multiconnected systems consists of a set of identical separate and mutual connections between single subsystems (Fig.1).

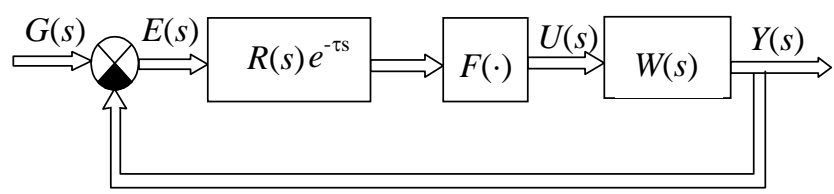

Fig. 1. The nonlinear structure of the same type, MSAC with delay

For systems with nonlinearities in direct channels of separate subsystems as a local characteristic of the harmonically linearized i-th subsystem, you can consider its transfer function $\Phi \mathrm{i}(\mathrm{s}, \mathrm{a})$ in control mode [3]:

$$
\Phi_{i}(s, a)=\frac{W_{H i}\left[q_{i}(a), q_{i}^{\prime}(a)\right] W_{i i}(s) e^{-\tau s}}{1+W_{H i}\left[q_{i}(a), q_{i}^{\prime}(a)\right] W_{i i}(s) e^{-\tau s}}
$$

where $W_{H i}\left[q_{i}(a), q_{i}^{\prime}(a)\right] W_{i i}(s) e^{-\tau s}-$ transfer function of harmonically linearized nonlinear element of $i$-th separate subsystem; $q_{i}(a), q_{i}^{\prime}(a)$ - coefficients of harmonic linearization; $W_{i i}(s) e^{-\tau s}$ - transfer functions of linear multidimensional object with delay $\tau$ along the i-th channel and the controller of the i-th separate subsystem. For the class of nonlinear same MSAC the hypothesis of identity of dynamic characteristics of harmonically linearized 
subsystems based on the content of identical nonlinear elements in its composition is valid [4]:

$$
\Phi_{1}\left[q_{1}(a), q_{1}^{\prime}(a), s\right]=\Phi_{2}\left[q_{2}(a), q_{2}^{\prime}(a), s\right]=\ldots=\Phi\left[q(a), q^{\prime}(a), s\right] .
$$

The characteristic equation of harmonically linearized the same MSAC can be written in the next form (2):

$$
\begin{aligned}
& 1+h_{2} \Phi^{2}\left[q(a), q^{\prime}(a), s\right]+h_{3} \Phi^{3}\left[q(a), q^{\prime}(a), s\right]+\ldots+ \\
& +h_{n} \Phi^{n}\left[q(a), q^{\prime}(a), s\right]=0
\end{aligned}
$$

where $h_{i}(i=\overline{2, n})$ - generalized numerical characteristics of multidimensional elements of communication between separate subsystems, calculated by the formulas proposed in $[4,5]$.

We introduce an algebraic equation of connection with respect to some variable $x=\Phi\left[q(a), q^{\prime}(a), s\right]$, obtained from (2)

$$
1+h_{2} x^{2}+h_{3} x^{3}+\ldots++h_{n} x^{n}=0
$$

$\left\{x_{i}\right\}$ - the set of value of equation (3), where $i=1,2 \ldots n$.

By frequency criterion [5]: the condition for the presence of periodic movements (PM) in this system is the passage of the local amplitude-phase frequency response (APF) $\Phi(a, j \omega)$ harmonically linearized subsystem through one value of the roots of the set $\left\{x_{i}\right\}$, which play the role of critical points $[6,7]$.

If you change the $0<a<\Delta$ deformed form of the hodograph APF $\Phi(a, j \omega)$. In general case, the critical point is the own value $\mathrm{x}_{\mathrm{i}}^{*}=\alpha_{\mathrm{i}} \mathrm{j} \beta_{\mathrm{i}}$, which go through the APF $\Phi(a, j \omega)$. Then equality is true:

$$
\Phi_{i}(a, j \omega)=\frac{W_{\mu i}\left[q_{i}(a), q_{i}^{\prime}(a)\right] W_{i i}(s) e^{-\tau j \omega}}{1+W_{H i}\left[q_{i}(a), q_{i}^{\prime}(a)\right] W_{i i}(s) e^{-\tau j \omega}}=x_{i}^{*}
$$

Consider the homogeneous nonlinear systems with delay in the cross-ties. Then, in the characteristic equation (2) for a complete ACS consisting of $n$ subsystems, the characteristic of the connection in the general form between $k$ subsystems has the form:

$$
\begin{gathered}
h_{k}^{*}(s)=\frac{\operatorname{det}\left[W_{i j}(s) \gamma_{i j}\right]_{k \times k}}{\operatorname{det}\left[W_{i j}(s) \delta_{i j}\right]_{k \times k}}, \gamma_{i j}=\left\{\begin{array}{l}
0, i=j, \\
e^{-\tau s}, i \neq j,
\end{array} ; \delta_{i j}=\left\{\begin{array}{l}
1, i=j, \\
0, i \neq j,
\end{array}\right.\right. \\
k=\overline{2, n} ; i=\overline{1, n}, \quad j=\overline{1, n},
\end{gathered}
$$

and local characteristic of the harmonically linearized $i$-th subsystem $\Phi_{i}(s, a)=\frac{W_{\mathrm{н} i}(a) W_{i i}(s)}{1+W_{\mathrm{н} i}(a) W_{i i}(s)}$. The presence of PM in a nonlinear ACS with a delay in cross-linking the fulfillment of the condition:

$$
\Phi_{i}(s, a)=\frac{W_{\mu i}(a) W_{i i}(s)}{1+W_{H i}(a) W_{i i}(s)}=x_{i}^{*}(s, \tau)
$$

So, on the basis of equality (4), (5) it is possible to using frequency methods, to determine the parameters of periodic motion in nonlinear one-type MSAC and to evaluate their stability.

\section{DETERMINATION OF PARAMETERS OF PERIODIC MOVEMENTS}

Determine parameters, amplitude $a^{*}$ and frequency $\omega_{i}{ }^{*}$, periodic movements. We distinguish the linear and nonlinear characteristics of the open subsystem from equation (4) [8]:

$$
W_{H}(a) W(j \omega) e^{-\tau j \omega}=\frac{\Phi(a, j \omega)}{1-\Phi(a, j \omega)}
$$

where $W_{H}(a)=q(a)+j q^{\prime}(a)$.

$W_{3}(j \omega=) W(j \omega) e^{-\tau j \omega}=(U(\omega)+j V(\omega))(\cos \omega \tau-j \sin \omega \tau)=$ $=(U(\omega) \cos \omega \tau-V(\omega) \sin \omega \tau)+j(V(\omega) \cos \omega \tau-U(\omega) \sin \omega \tau)=$ $=U_{3}(\omega)+j V_{3}(\omega)$.

Substitute $x_{i}^{*}$ instead $\Phi(a, j \omega)$ in the equation (6):

$$
W_{H}(j \omega) W_{3}(j \omega)=\frac{x_{i}^{*}}{1-x_{i}^{*}}=c_{i}+j d_{i} .
$$

To solve the equation (7), we select the linear parts of the system and use two scalar equations:

$$
\left\{\begin{array}{l}
\left|W_{3}(j \omega)\right|=\frac{\left|c_{i}+j d_{i}\right|}{\left|W_{H}(a)\right|} ; \\
\arg W_{3}(j \omega)=\arg \left(c_{i}+j d_{i}\right)-\arg W_{H}(a) .
\end{array}\right.
$$

or we can wrte:

$$
\left\{\begin{array}{l}
\sqrt{\left.U_{3}^{2}(\omega)+V_{3}^{2} \omega\right)}=\frac{\sqrt{c_{i}^{2}+d_{i}^{2}}}{\sqrt{q^{2}(a)+\left[q^{\prime}(a)\right]^{2}}} ; \\
\operatorname{arctg} \frac{V_{l}(\omega)}{U_{l}(\omega)}-\operatorname{arctg}(\omega \tau)=\operatorname{arctg}\left(\frac{d_{i}}{c_{i}}\right)-\operatorname{arctg}\left(\frac{q^{\prime}(a)}{q(a)}\right) .
\end{array}\right.
$$

After solving a system of two equations, we find the values $a^{*}$ and $\omega$

\section{STABILITY ANALYSIS OF PERIODIC MOVEMENTS IN NONLINEAR MCACS}

To determine the parameters of PD and analyze their stability, you can use the graph-analytical method, consisting of the following steps:

1. Find the roots $x_{i}$ of the equation of communication (3);

2. From equation (7) we distinguish the linear and nonlinear parts of the system:

$$
W_{3}(j \omega)=Z_{H}^{*}(j a) \text {, where } Z_{H}^{*}(j a)=\frac{1}{W_{H}(a)} \frac{x_{i}^{*}}{1-x_{i}^{*}}=\frac{c_{i}+j d_{i}}{W_{H}(a)} \text {. }
$$

3. Build on the complex plane $W_{3}(j \omega)$ and $Z_{H}^{*}(j a)$;

4. We define the parameters $a^{*}$ and $\omega^{*}$ periodic movements at the intersection of the characteristics $W_{3}(j \omega)$ and $Z_{H}^{*}(j a)$; 
5. To evaluate the stability of periodic movements by the frequency criterion [5].

Periodic movements in a nonlinear homogeneous MCACS will be stable if the amplitude $a^{*}$ by $\Delta a$ increases (decreases) at the point corresponding to the intersection of hodographs $W_{3}(j \omega)$ and $Z_{H}^{*}(j a)$, APF $W_{3}(j \omega)$ does not cover a new point $a=a^{*}+\Delta a$, otherwise, PM will be unstable [9-11].

Example 1. We investigate a two-connected system (Fig. 1) for the presence of self-oscillations in the presence of a nonlinear element of the "saturation" type (Fig. 2) and delays in subsystems.

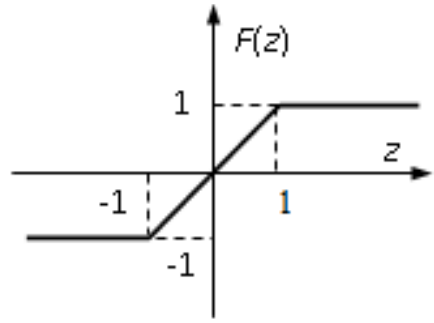

Fig. 2. The nonlinear element of the type "saturation"

The coefficients of harmonic linearization for the characterization of $F(z)$ of the type "saturation" is equal to:

$q(a)=\frac{2 k}{\pi}\left(\arcsin \frac{b}{a}+\frac{b}{a} \sqrt{1-\frac{b^{2}}{a^{2}}}\right) \quad$ by $a \geq b ; b=1$

$q(a)=k \quad$ by $a<b$;

$q^{\prime}(a)=0$.

Transfer function of a two-dimensional object with delay:

$W_{i j}(s)=\frac{e^{-\tau s}}{\mathrm{~s}(\mathrm{~s}+1)}\left[\begin{array}{cc}1 & -2 \\ 2 & 1\end{array}\right], i=j=1,2 ; \tau=5$.

Consider the solution of this problem in stages.

1. Transfer functions $\Phi_{\mathrm{i}}(s, a), i=1,2$ separate channels in control mode:

$\Phi(a, s)=\frac{W_{H}(a) W(s) e^{-\tau s}}{1+W_{H} W(s) e^{-\tau s}}=\frac{q(a) e^{-\tau s}}{s^{2}+s+q(a) e^{-\tau s}}$.

Characteristic equation of the system:

$1+h_{2} \Phi(a, j \omega)^{2}=0$, where $h_{2}=4$.

Coupling equation: $1+4 x^{2}=0$.

The roots of the coupling equation are $x_{1}=+0.5, x_{2}=-0.5$.

2. Select the linear and nonlinear parts of the system:

$W_{3}(j \omega)=\frac{e^{-\tau j \omega}}{(j \omega)^{2}+j \omega} ;$

$Z_{\mathrm{H}}^{*}(j a)=\frac{x_{2}}{1-x_{2}} \cdot \frac{1}{W_{H}(j a)}=\frac{-0.2-0.4 j}{q(a)}$.

3. We create hodographs on the complex plane $W_{3}(j \omega)$ and $Z_{H}^{*}(j a)$ (fig. 3).
4. Figure 3 shows that PM is present in the system. At point 1 the hodographs intersect $W_{3}(j \omega)$ and $Z_{H}^{*}(j a)$ at delay $\tau=5$ with parameters PM $a=11.8, \omega=0.23$ (fig. 4), at point 2 when the delay $\tau=0$ with the parameters PM a=4.9, $\omega=0.5$. (fig. 5).

5. We estimate the stability of periodic motions by the frequency criterion [5].

Periodic motion in the system is stable, because with increasing amplitude $a^{*} \Delta a$ in points 1 and 2 corresponding to the intersection of hodographs $W_{3}(j \omega) W(j \omega)$ locus $Z_{\mathrm{H}} *(j a)$, APF $W_{3}(j \omega) W(j \omega)$ do not cover the new point $a=a^{*}+\Delta a$.

The results confirm the transients (Fig. 4, 5), obtained by modeling the original system.

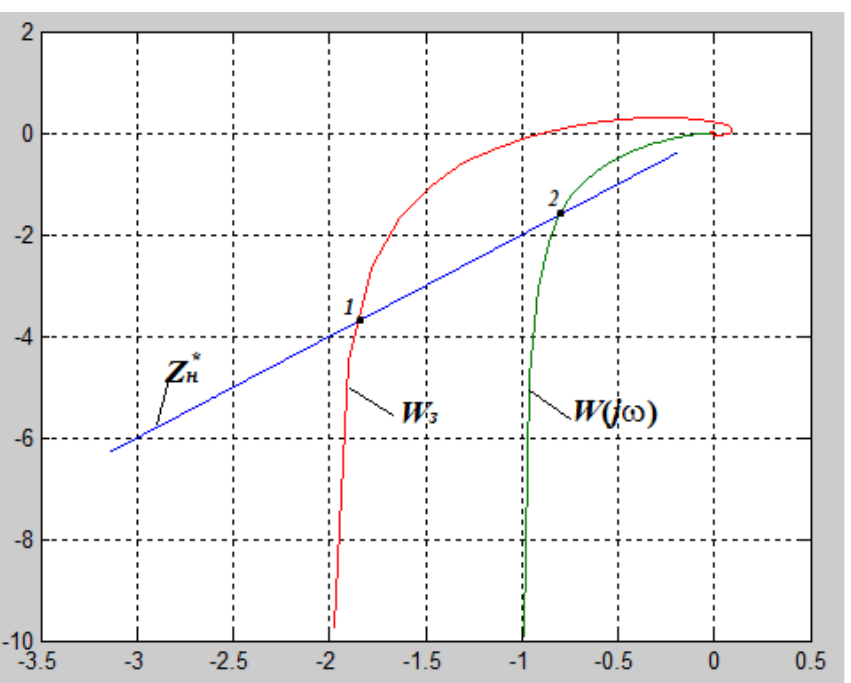

Fig. 3. The intersection of hodographs $W_{3}(j \omega) W(j \omega)$ locus $Z_{\mathrm{H}} *(j a)$



Fig. 4. PM in the system with delay (point 1 ) with parameters $a=4.9$, $\omega=0.5$. 


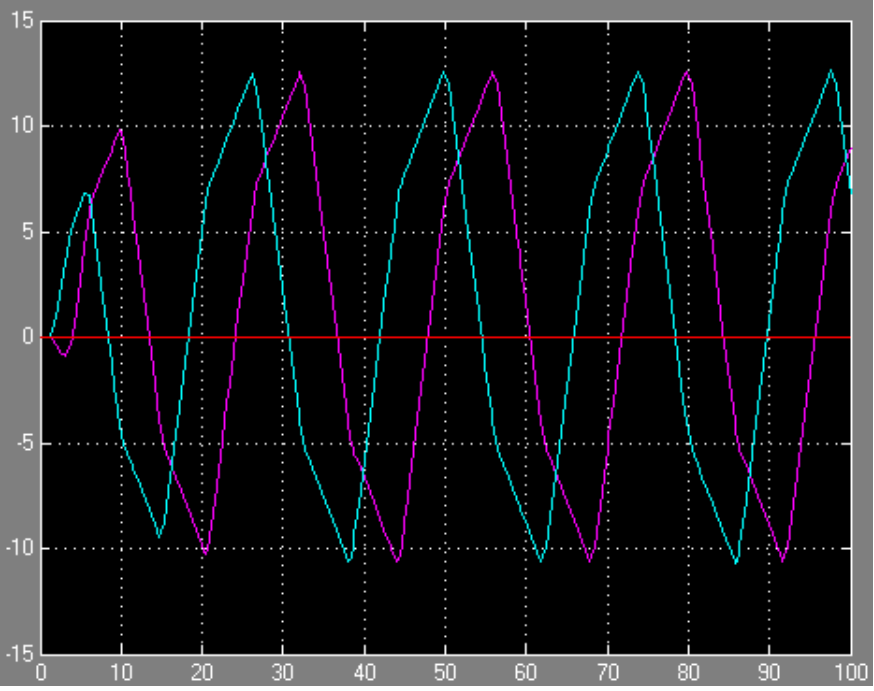

Fig. 5. PM in the system with delay (point 2) with parameters $a=11.8$, $\omega=\mathbf{0 . 2 3}$.

To determine the parameters of PM and analyze their stability in a nonlinear ACS with delay in cross-links, consider the next steps:

1. Find the root $x_{i}^{*}(j \omega, \tau)$ of the hodograph equation $(3)$

2. From equation (7) we select the linear and nonlinear parts of the system:

$$
W_{ת}(j \omega)=Z_{H}^{*}(j a)
$$

where $W_{\Omega}(j \omega)=W(j \omega)=\frac{1-x_{i}^{*}(j \omega, \tau)}{x_{i}^{*}(j \omega, \tau)}, Z_{H}^{*}=\frac{1}{W_{H}(a)}$.

3. We create on the complex plane $W_{\Omega}(j \omega), Z_{H}^{*}(j a)$;

4. We define the parameters $a^{*}$ and $\omega^{*}$ periodic movements at the intersection of the characteristics $W_{l}(j \omega)$ and $Z_{H}^{*}(j a)$;

5. To evaluate the stability of periodic movements by the frequency criterion [5].

Example 2. Investigate the two-connected system (Fig. 1) with nonlinear elements of the "saturation" type in subsystems (Fig. 2, example 1) and the delay in cross-linking for the presence of self-oscillations.

Transfer function of a two-dimensional object with delay:

$$
W_{i j}(s)=\frac{1}{s(s+1)}\left[\begin{array}{cc}
1 & -2 e^{-\tau s} \\
2 e^{-\tau s} & 1
\end{array}\right], i=j=1,2 ; \tau=1 .
$$

Consider the solution of this problem in stages.

1. Transfer functions $\Phi_{i}(s, a), i=1,2$ separate channels in control mode:

$$
\Phi(a, s)=\frac{W_{H}(a) W(s)}{1+W_{H} W(s)}=\frac{q(a)}{s^{2}+s+q(a)} .
$$

2. Characteristic equation of the system (2), (6): $1+h_{2} e^{-2 \tau s} \Phi(a, j \omega)^{2}=0$, where $h_{2}=4$.

3. Coupling equation (3) for the determination of root hodographs: $1+h_{2} e^{-2 \tau s} x^{2}=0$.

4. Select the linear and nonlinear parts of the system:

$$
W_{n}(j \omega)=\frac{1}{(j \omega)^{2}+j \omega} * \frac{1-x_{i}^{*}(j \omega, \tau)}{x_{i}^{*}(j \omega, \tau)} ; Z_{H}^{*}=\frac{1}{W_{H}(a)}=\frac{1}{q(a)} .
$$

5. Create hodographs on the complex plane $W_{n}(j \omega)$ and $Z_{H}^{*}(j a)$ (fig. 6).

6. Define the parameters $a^{*}$ and $\omega^{*}$ periodic movements at the intersection of the characteristics $W_{л}(j \omega)$ and $Z_{H}^{*}(j a)$.

Figure 6 shows that PM present in the systems, and cross the hodographs $W_{\pi}(j \omega), Z_{H}^{*}(j a)$ at delay $\tau=1$ (Fig. 7). At delay $\tau=0$ (Fig. 6) no PM, because hodographs do not cross.

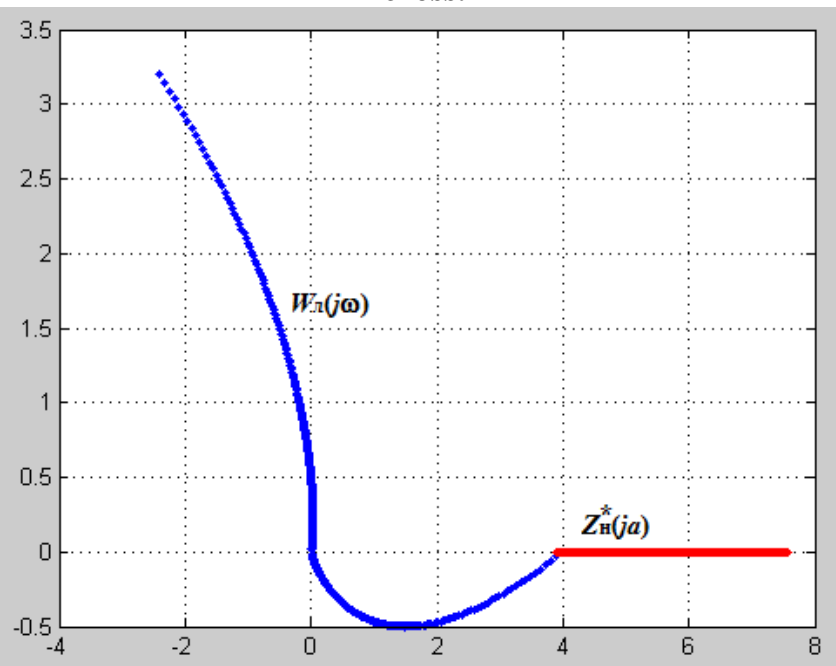

Fig. 6. The intersection of hodographs $W_{л}(j \omega)$ and $Z_{\mathrm{н}} *(j a)$ without delay Parameters $a=4.9, \omega=0.5, \tau=0$.

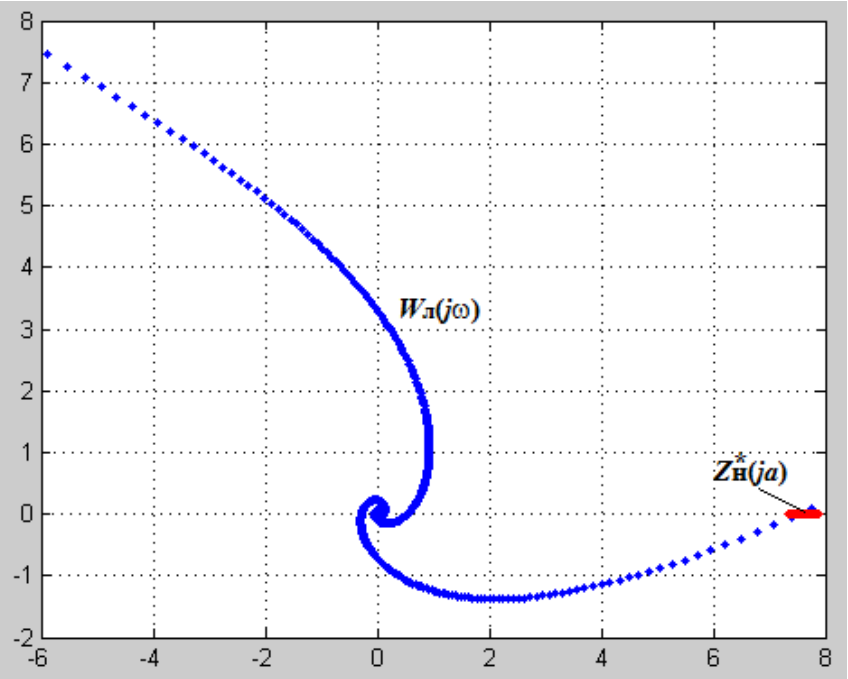

Fig. 7. The intersection of hodographs $W_{\mathrm{л}}(j \omega)$ and $Z_{\mathrm{r}} *(j a)$ with delay $\tau=1$

Parameters $a=9.3, \omega=0.27, \tau=1$ 
7. Assessing the stability of periodic movements on the frequency criterion it can be concluded, that in the presence of delay in the cross-links PM stable.

The results confirm the transition processes (Fig. 8, 9), obtained by modeling the original system.

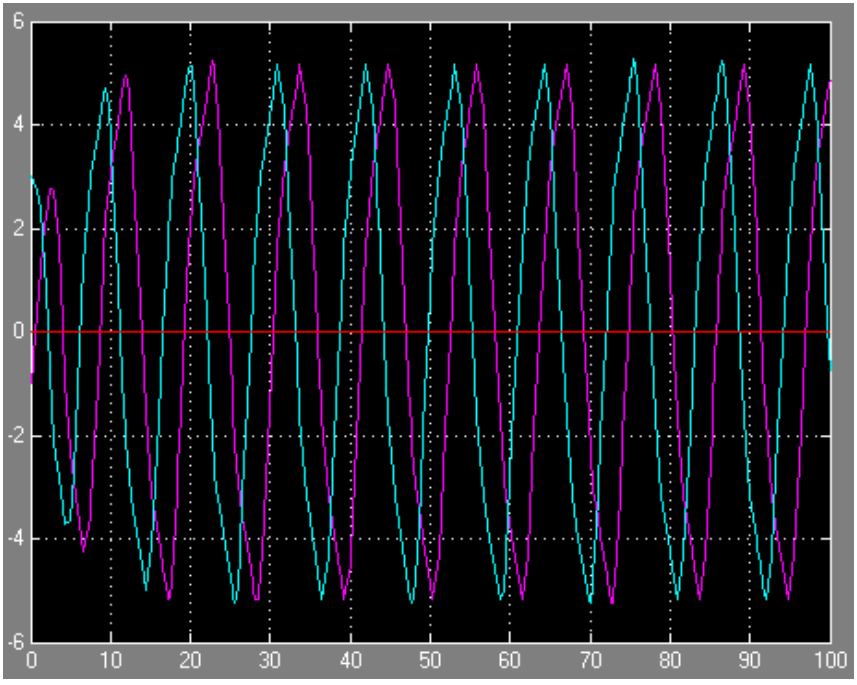

Fig. 8. $P M$ in the system without delay $\tau=0$ with parameters $a=4.9, \omega=0.5$

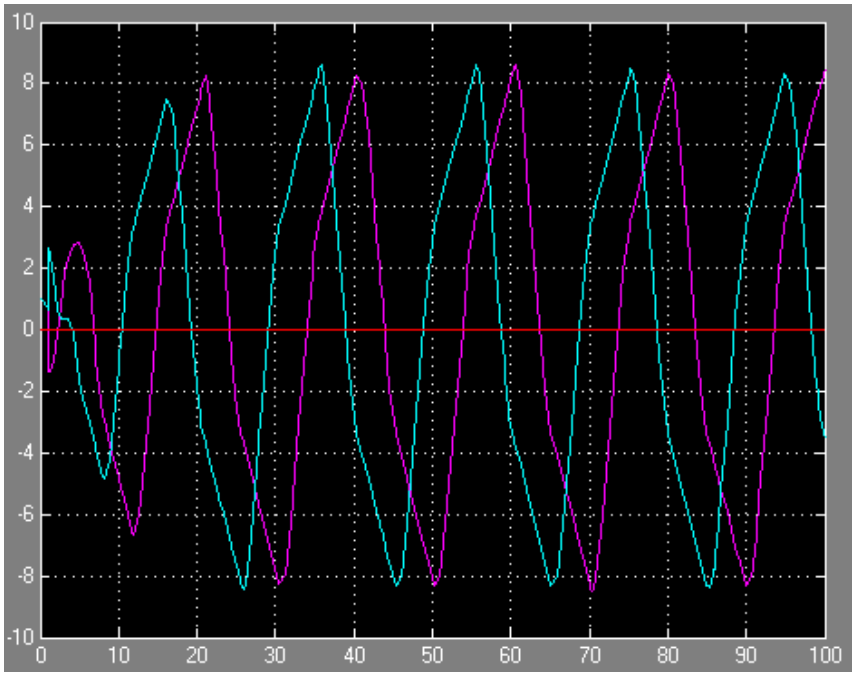

Fig. 9. $P M$ in the system with delay $\tau=1$ with parameters $a=8.3, \omega=0.3$

In the study of systems with delay is obtained of intersection of hodographs $W_{l}(j \omega)$ and $Z_{H}^{*}(j a)$ (fig. 7). The amplitude and frequency are approximately the same as the results of the simulation (fig. 7,9).

Thus, the paper proposes an approach based on frequency methods and the harmonic linearization method, which allows to estimate the parameters and stability of periodic motions in the same type nonlinear MSAC with nonlinearities and delay in direct channels and crosslinks[12,13].

\section{CONCLUSION}

As a result of the study, a nonlinear multi-connected system of automatic control of the object with delay (control and output) by frequency methods, based on the harmonic linearization method and the system description of the MSAC characteristics was studied. The ACS with a nonlinear element and delay in direct channels and crosslinks is considered, provided that this ACS consists of a set of identical separate subsystems, the mutual relations between the subsystems are single. Used frequency-domain methods for the parameters of the PM to nonlinear similar MSAC, and assessed their stability. The results are confirmed by simulation in Matlab Simulink package.

\section{ACKNOWLEDGMENT}

The work was supported by the Russian Foundation for basic research (RFFI Grants №18-08-00702 A, 17-48020956 p_a).

\section{REFERENCES}

[1] A. B. Filimonov, Spectral decomposition of systems with delays. Compensation for delays. M: Phyz.-Math. Lit-ry, 2002, in Russian.

[2] B. G. Ilyasov, Y. S. Kabalnov, "Investigation of the stability of homogeneous multiply connected automatic control systems with homonymy relationships between sub-systems" in Automatics and telemechanics, vol 7. Road town: Pleiades Publishing, Ltd, 1995, in Russian, pp. 82-90.

[3] V. A.Besekersky, E. P. Popov, Theory of automatic control systems. SPb; Publishing house "Profession", 2014, in Russian.

[4] D. P. Kim, Theory of automatic control. Volume 2. Multidimensional, nonlinear, optimal and adaptive systems. M.: Phyz.-Math, 2016, in Russian.

[5] B. G. Ilyasov, G. A. Saitova, "A systems approach to studying multi-connected automated control systems based on frequency methods" in Automation and Remote Control, vol.3. Moscow: Russian Academy of science, 2013, pp. 456-470.

[6] B. G. Ilyasov, G. A. Saitova, "Stability analysis of dynamic systems in polynomial vector-matrix representation" in Journal of Computer and Systems Science International, vol. 2. Road town: Pleiades Publishing, Ltd, 2018, pp. 171-178.

[7] I. Kudinov, Theory of automatic control (using the MATLAB - SIMULINK). SPb.: DOE, 2016, in Russian.

[8] A. A. Erofeev, Theory of automatic control: textbook for universities,SPb.: Polytechnic, 2008, in Russian.

[9] B.G .Ilyasov, G.A. Saitova, E.A. Khalikova, "The synthesis of algorithms for intelligent control of gascompressor unit to ensure the required quality" in Modern top technologies, №12, vol. 2, Penza: Publish. House "Academy of natural sciences", 2018, in Russian, pp. 271-275.

[10] B.G. Ilyasov, G.A. Saitova, E.A. Khalikova, "Analysis of the stability margins of homogeneous multivariable control systems" in Journal of Computer and Systems Sciences International, vol. 4, Road town: Pleiades Publishing, Ltd, 2009, pp. 502-510.

[11] B.G. Ilyasov, G.A. Saitova, E.A. Khalikova, "Program system for designing of multivariable control system for complex objects" in Computer science and information technologies (CSIT'2009), vol. 1, Crete, Greece: proceedings of the 11th International Workshop, 2009, in Russian, pp.96-100.

[12] O.D. Lyantsev, G.G. Kulikov, V.Y. Arkov, T.V. Breikin, "Optimal multi-variable control of gas turbine engines" in International Journal of Systems Science, Taylor\&Francis, vol. 35, №2. 2004, pp. 79-86. 
[13] N. Xavier, B. Bandyopadhyay, R. Schmid, "Robust nonovershooting tracking using continuous control for linear multivariable systems" in IET Control Theory and Applications, vol. 12, №7, 2018, pp. 1006-1011.

[14] C. Chen, Z. Liu, K. Xie, Y. Zhang, C. Wen, C.L.P. Chen, "Adaptive asymptotic control of multivariable systems based on a one-parameter estimation approach", vol. 83 :Automatica, 2017, pp. 124-132.

[15] G. Alevisakis, D.E. Seborg, "An extension of the Smith predictor method to multi-variable linear system containing time delays”, Int. J. Contr., vol. 17, №3, 1973, pp. 541-551.

[16] Qing-Chang Zhong, "Robust Control of time-delay systems", Springer-Verlag: London Limited, 2006, pp. 420-457.
[17] A.Z. Manitius, A.W. Olbrot, "Finite spectrum assignment problem for systems with delays" in IEEE Trans. Automat. Contr., №4, 1979, pp.541-553.

[18] Qing-Chang Zhong, "Robust Control of time-delay systems", Springer-Verlag London Limited, 2006, pp. 420-457.

[19] A. Balestrino, F.B. Verona, A. Landi, "On-line process estimation by ANNs and Smith controller design", IEE Proc.-Control Theory Appl., vol. 145, №2, 2008, pp. 231-235.

[20] A.Z. Manitius, A.W. Olbrot, "Finite spectrum assignment problem for systems with delays", IEEE Trans. Automat. Contr., №4, 2009, pp.541-553. 